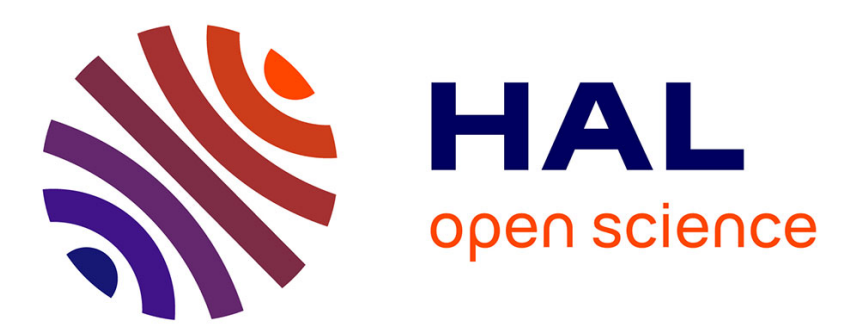

\title{
Kinetic Monte Carlo simulation of random deposition and scaling behavior with respect to the germination length
}

\author{
Abdenour Saoudi, Linda Aissani, Grégoire Sorba, Francisco Chinesta
}

\section{To cite this version:}

Abdenour Saoudi, Linda Aissani, Grégoire Sorba, Francisco Chinesta. Kinetic Monte Carlo simulation of random deposition and scaling behavior with respect to the germination length. International Journal of Computational Materials Science and Engineering, 2021, 09 (04), pp.2050022. $10.1142 / \mathrm{s} 2047684120500220$. hal-03219648

\section{HAL Id: hal-03219648 \\ https://hal.science/hal-03219648}

Submitted on 6 May 2021

HAL is a multi-disciplinary open access archive for the deposit and dissemination of scientific research documents, whether they are published or not. The documents may come from teaching and research institutions in France or abroad, or from public or private research centers.
L'archive ouverte pluridisciplinaire HAL, est destinée au dépôt et à la diffusion de documents scientifiques de niveau recherche, publiés ou non, émanant des établissements d'enseignement et de recherche français ou étrangers, des laboratoires publics ou privés. 


\title{
Kinetic Monte Carlo simulation of random deposition and scaling behavior with respect to the germination length
}

\author{
Abdenour Saoudi \\ Mechanical Engineering Department \\ Abbes Laghrour-Khenchela University \\ P.O. Box 1252, Khenchela 40004, Algeria \\ Linda Aissani \\ Laboratory of Active Components and Materials \\ Larbi Ben M'Hidi University, Oum El Bouaghi, Algeria \\ Mater Science Department, Abbes Laghrour-Khenchela University \\ P.O. Box 1252, Khenchela 40004, Algeria \\ Grégoire Sorba \\ École Supérieure des Techniques Aéronautiques et Construction Automobile \\ 12 Avenue Paul Delouvrier, 78180 Montigny-le-Bretonneux, France \\ Francisco Chinesta* \\ PIMM Lab \& ESI Chair, Arts et Metiers Institute of Technology \\ 151 Boulevard de l'Hôpital, 73013 Paris, France \\ francisco.chinesta@ensam.eu
}

\begin{abstract}
This work aims at analyzing the scaling behavior and develop correlations during surface growing for different germination lengths. The surface growing by random deposition is simulated using a kinetic Monte Carlo approach, by considering different germination lengths. Different surface descriptors are extracted, among them the roughness and the correlation. The former allows extracting the scaling behavior, while the latter proves the existence of correlations independent of the system size but dependent on the germination length. Moreover, as in the case of random deposition with a null germination length, the growing roughness never saturates.
\end{abstract}

Keywords: Random deposition; germination length; roughness; growth scaling.

\section{Introduction}

Functional coatings are nowadays major players empowering high-technology applications, as the ones concerning functional surfaces enabling oxidation resistance or 
anti-condensation, among many others. These functional surfaces are elaborated by using physical vapor deposition (PVD) and chemical vapor deposition (CVD), with the surface growing due to the continuous atoms bombardment that the surface experiences.

In this context, it is necessary to have a tool able to predict the growth evolution of the film. Traditionally, two main mathematical descriptions were widely employed for describing the evolving surface morphology: (i) the kinetic Monte Carlo (kMC) simulations [Family and Vicsek, 1991; Halpin-Heraly and Zhang, 1995; Duke and Plummer, 2002; Das Sarma and Tamborenea, 1991; Barato et al., 2008; Albano et al., 1999; Forgerini and Figueiredo, 2009, 2011; Cansizoglu et al., 2015], based on tracking a huge number of particles obeying quite simple microscopic rules and (ii) the stochastic differential equations (SDEs) [Haselwandter and Vvedensky, 2007; Majaniemi et al., 1996].

kMC simulations combine the simplicity of its computational implementation with their deep physical content. However, kMC-based approaches do not allow to obtain closed forms of the solution, making difficult the implementation of optimization or inverse analyses. On the other hand, SDE allows a more rigorous solution procedure, enabling its employment in controllers, however, the modeling stage represents its trickiest issue, where usually a number of hypotheses must be considered for deriving a closed model. This paper will focus on the former approach, the kMC.

When using the kMC approach, the behavior of an atom reaching a surface will depend on the energy of the atom, the atom-surface interaction (chemical bonding) and the temperature of the surface. The mobility on a surface can vary depending on the chemistry, crystallography, etc. affecting the surface diffusion.

Atoms condense on a surface by losing energy by creating and breaking chemical bonds with the substrate atoms, colliding with other diffusing surface atoms, finding preferential nucleation sites and colliding or reacting with adsorbed surface species.

When atoms condense, they form nuclei. According to the nature of interaction between the deposited atoms and the substrate material it is possible to identify three types of growing mechanisms: (i) layer-by-layer growth; (ii) island growth, characterized by a three-dimensional nucleation and growth and (iii) combining layer and island growth.

One of the descriptors used to study surface growing and roughening processes is scaling. It is well established that some characteristics of the growing surfaces present scale-invariant properties, so that quite different growing processes exhibit very similar scaling behaviors. The time evolution of a surface induced by the deposition of particles is usually described in terms of some scaling exponents. These scaling exponents define the most fundamental characteristics of the surface growth, and allows to consider different processes belonging to the same universal class.

In many circumstances, the roughness increases exhibiting saturation after a certain time. This time as well as the resulting roughness can depend on the system size, proving that the saturation phenomenon involves a finite length effect. These effects result from physical correlations. 
During the growth process, correlations develop along the surface, which imply that the different sites of the surface are not totally independent, but depend on the heights of neighboring sites, conferring a nonlocality to the growth process. The correlation length determines the length in which the roughness influences the growth of a given site. At the beginning of the process, the locations where particles are deposited remain too far (with respect to the correlation length) and consequently the growing dynamics seems uncorrelated, fully local and without length effects. Correlations appear later.

This work aims at analyzing the effect of microscopic deposition physical rules, in particular the germination length, on the scaling exponents describing the growing dynamics.

\subsection{Surface descriptors and scaling exponents}

Scaling theory describes the dynamics of rough surfaces, in particular the standard deviation of time-dependent height [Family, 1986; Meakin, 1985], describing the surface roughness $W$

$$
W(t, L)=\sqrt{\frac{1}{L} \sum_{i=1}^{L}(h(t, i)-\bar{h}(t, L))^{2}},
$$

where $L$ is the size of the system, $h(t, i)$ is the height at the site $i$ on the surface at instant $t$ and $\bar{h}$ is the mean value of the height at that instant, given by

$$
\bar{h}(t, L)=\frac{1}{L} \sum_{i=1}^{L} h(t, i) .
$$

Generally, the surface roughness increases as a power of time until a time $t_{x}$, called crossover time [Mal et al., 2011], according to

$$
W(t, L) \sim t^{\beta}, \quad t \ll t_{x},
$$

where $\beta$ is the so-called growth exponent.

The crossover time represents the time required to change the growing regime and that depends on the system size according to the power law

$$
t_{x} \sim L^{z}
$$

where $z$ is the so-called dynamic exponent. After the crossover time $t_{x}$, the regime can turn into a saturation regime, due to correlations occurring on the surface, regime in which the roughness reaches a saturation value that remains constant in time but increases as $L$ increases, according to

$$
W_{\text {sat }}(L) \sim L^{\alpha}
$$

where $\alpha$ is the so-called roughness exponent.

The relation between the exponents depends on the growth process and has been widely studied [Coy and Sidik, 1985; Meakin, 1993]. The exponents $\alpha, \beta$ and 
$z$ characterize the growth of surfaces and their structural properties (self-affine, selfsimilarity, fractal dimension, etc.) [Barabasi et al., 1995; Forgerini and Figueiredo, 2011].

With respect to critical phenomena, only two exponents are needed to characterize the dynamics and consequently to define universal classes of rough surfaces [Binder, 1976].

Another usual procedure consists of studying surface correlations [Saoudi et al., 2017; Mirabella and Aldao, 2016], with the height correlation function calculated from

$$
C(x, t)=\left\langle\left(h\left(x_{0}+x, t\right)-h\left(x_{0}, t\right)\right)^{2}\right\rangle_{x_{0}} .
$$

\section{Growth Model}

In the random deposition addressed in this work, taking place on a surface of length $L$ (corresponding to the number of sites on the surface at which the particles reaching the surface can attach), a number of particles $N$ large enough (e.g., $N=10 \times L)$ are randomly projected on the surface. When one particle reaches the surface at site $i \in[1, \ldots, N]$ (where periodicity is assumed, i.e., $N+1 \equiv 1$ and $0 \equiv N$ ), its final location is given by some microscopic rules. The ones here considered, for a given germination length $l_{G}$, are as follows:

- If $h_{i}<\max \left\{h_{j}, j \in\left[i-l_{G}, i+l_{G}\right]\right\}$, then the particle will move to the site $k$ of maximum height, $h_{k}=\max \left\{h_{j}, j \in\left[i-l_{G}, i+l_{G}\right]\right\}$ with $80 \%$ of probability or stay at site $i$ with the remaining $20 \%$ probability. It they are different sites in that interval with the same (maximum) height, the $80 \%$ probability is equally distributed between those sites.

- If $h_{i}=\max \left\{h_{j}, j \in\left[i-l_{G}, i+l_{G}\right]\right\}$, then the particles attach to site $i$ with $80 \%$ probability or move to the site of highest height when excluding one of the sites $i$, $h_{i}$, i.e., to site $k$, with $h_{k}=\max \left\{h_{j}, j \in\left[i-l_{G}, i+l_{G}\right], j \neq i\right\}$ with $20 \%$ probability.

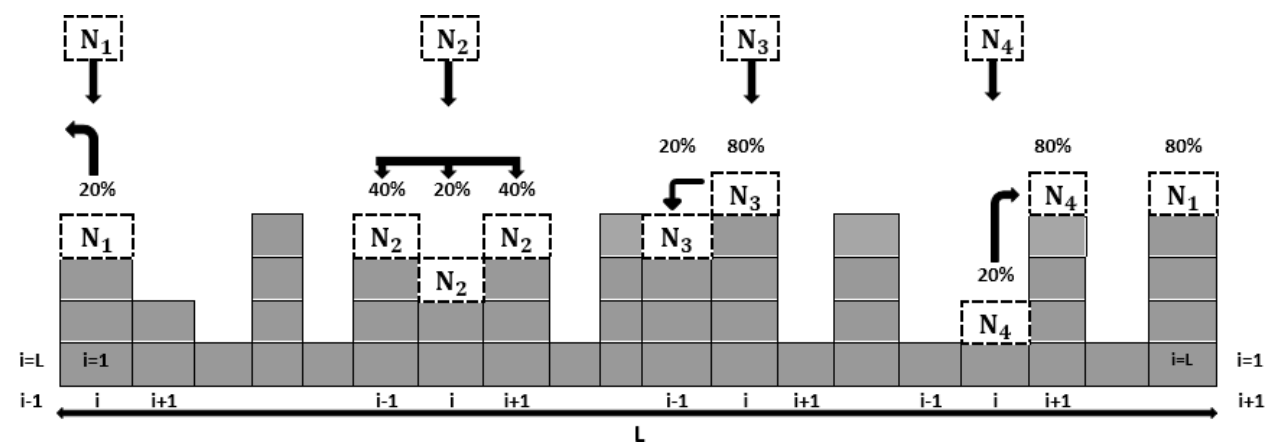

Fig. 1. Schematic diagram for some surface growth processes in the random deposition with a germination length $l_{G}=1$. 
It they are in different sites in that interval with the same (maximum) height, the $20 \%$ probability is equally distributed between those sites.

Figure 1 illustrates these microscopic rules for $l_{G}=1$.

\section{Scaling Behavior}

In order to optimize surface properties and performances, it is necessary to simulate the growth mechanism. The different growth stages are very influenced by the number of particles and the surface roughness [Petrov et al., 2003]. The germination effects emulate surface tension in a liquid surface, when addressing PVD [Jeong and Boo, 2004].

The surface roughness is expected depending on the germination length $l_{G}$ as the kMC simulations show in Fig. 2, as reported in Elsholz and Schöll [2004] when addressing the growth of $\mathrm{SiO}_{2}$ and $\mathrm{Nb}_{2} \mathrm{O}_{5}$ thin films.

The interface becomes less dense at higher germination lengths $l_{G}$, with particles concentrating in the highest surface peaks, such that the larger germination lengths are possible according to the growth model described in Sec. 2. Thus, the growth mainly localizes at some locations that attract high probability particles that reached the surface at neighboring positions (neighbors in the sense of the
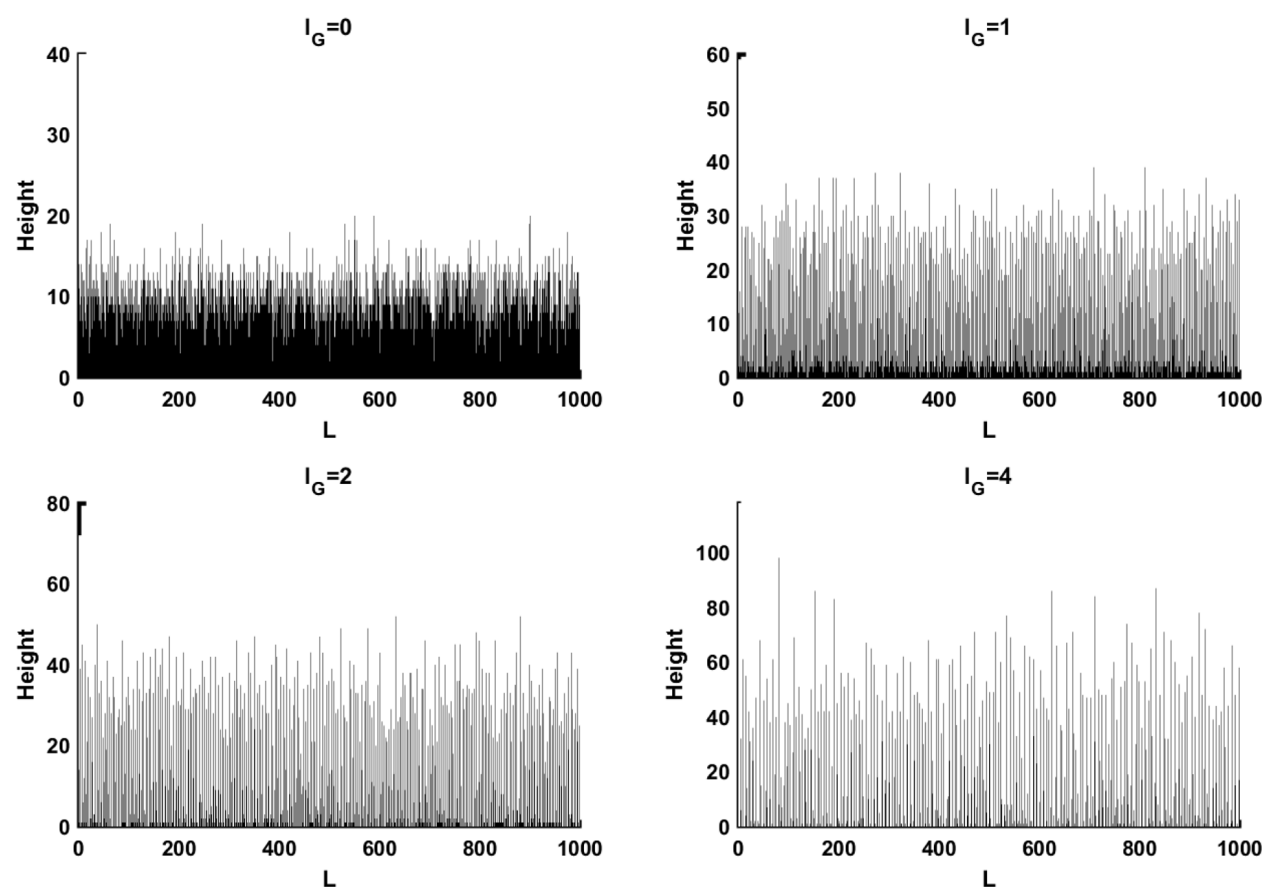

Fig. 2. Surface topography obtained for different germination lengths $l_{G}$ in random deposition. Both, the system size $L$ and the computational particles $N$, were kept constant, $L=1000$ and $N=10000$. 
germination length $l_{G}$ ). This mechanism seems in agreement with the observations reported in Raoufi and Hosseinpanahi [2013].

For different sizes of the system $L$ and the germination length $l_{G}$, two different growth regimes are noticed, as Figs. 3 and 4 evidence. An initial growing regime occurs when the number of deposited particles remains smaller than the system size $(L)$. In this regime, the germination length has a negligible effect, with the growth dynamics approaching a purely random deposition. Then, when the number of the particles becomes greater than the system length, the effects of the germination

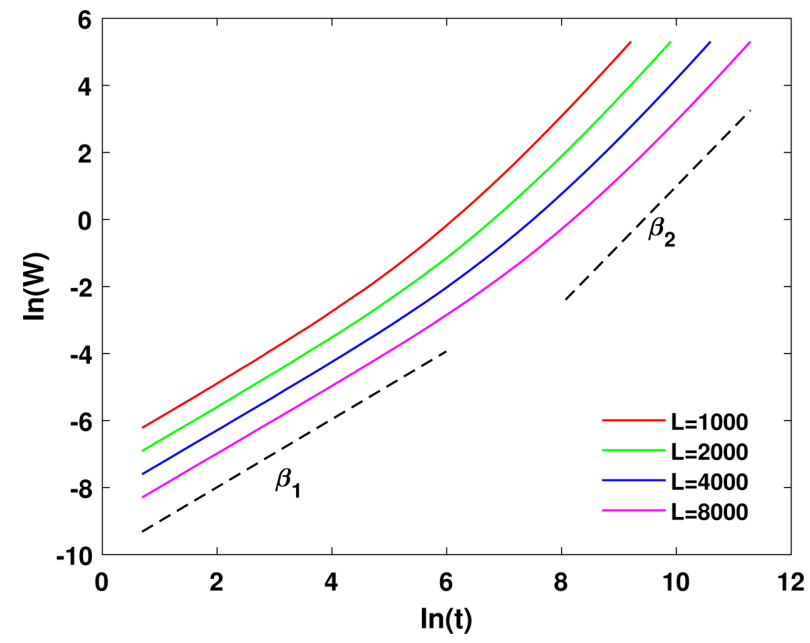

Fig. 3. Growing dynamics for $l_{G}=2$ and different system size $L$. Dashed lines represent the exponents $\beta_{1}$ and $\beta_{2}$ (averages are computed on 100 different configurations).

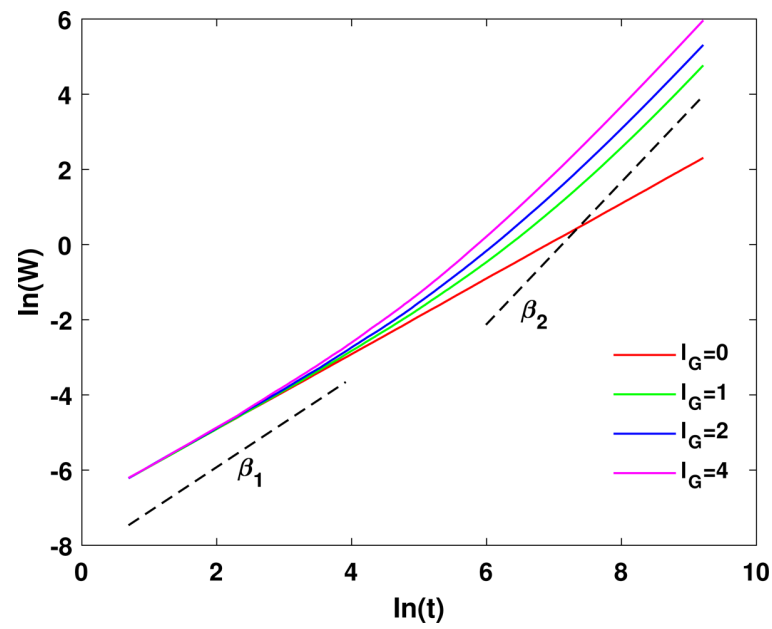

Fig. 4. Growing dynamics for $L=10000$ and different germination length $l_{G}$. Dashed lines represent the exponents $\beta_{1}$ and $\beta_{2}$ (averages are computed on 100 different configurations). 
length on the growth dynamics become preponderant, and a change in the slope is noticed. As expected, the growth strongly depends on the germination length $l_{G}$ in this terminal regime, with the growing exponent independent of the system size $L$.

The growth exponents $\beta_{1}$ and $\beta_{2}$ were calculated from the slope of the roughness $\operatorname{logarithm}(\ln (W))$ as a function of $\ln (t)$. As expected, the initial regime approaches the usual uncorrelated random deposition with null germination length, and its growth exponent $\beta_{1}=0.5055$ is in perfect agreement with the expected coefficient 0.5 .

The terminal regime is characterized by a greater growth exponent, $\beta_{2}=0.8982$ (for $l_{G}=2$ ), and as previously indicated, both $\beta_{1}$ and $\beta_{2}$ are independent of the system size $L$, but exhibit a significant (expected) dependence on the germination length, with $\beta_{2}$ differing from 0.5 when $l_{G}$ increases, as reported in Table 1.

The growing dynamics induced by the microscopic rules discussed previously avoids reaching a saturation regime.

Figures 5 and 6 show the evolution of the correlation according to expression (6) when varying the system size $(L)$ and the germination length $\left(l_{G}\right)$, respectively.

Table 1. Growth exponents $\beta_{1}$ and $\beta_{2}$ for random deposition with different germination length $l_{G}(L=10000)$.

\begin{tabular}{ccc}
\hline$l_{G}$ & $\beta_{1}$ & $\beta_{2}$ \\
\hline 0 & 0.5000 & 0.5000 \\
1 & 0.5019 & 0.8754 \\
2 & 0.5055 & 0.8982 \\
4 & 0.5135 & 0.9313 \\
\hline
\end{tabular}

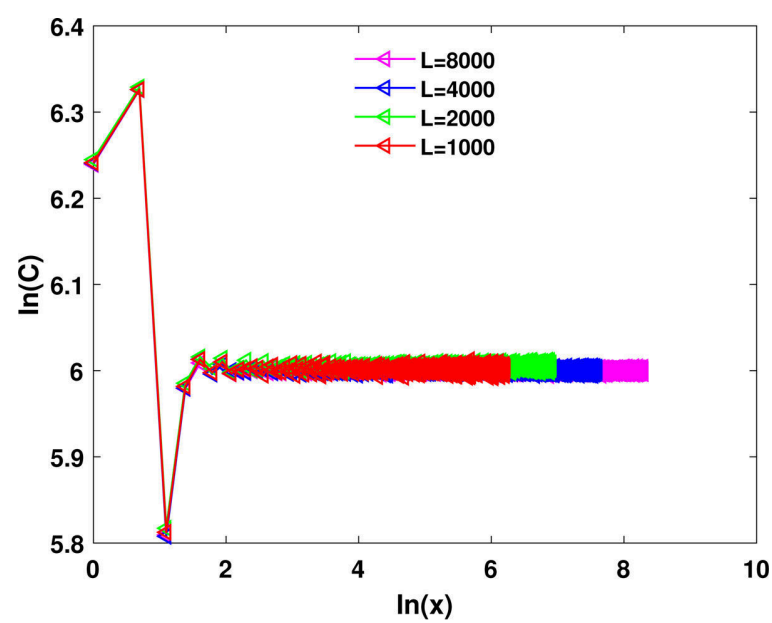

Fig. 5. Correlation in rough surfaces generated by random deposition with germination length $l_{G}=2$ for different system sizes $L$ (averages are computed over $k=100$ different configurations). 


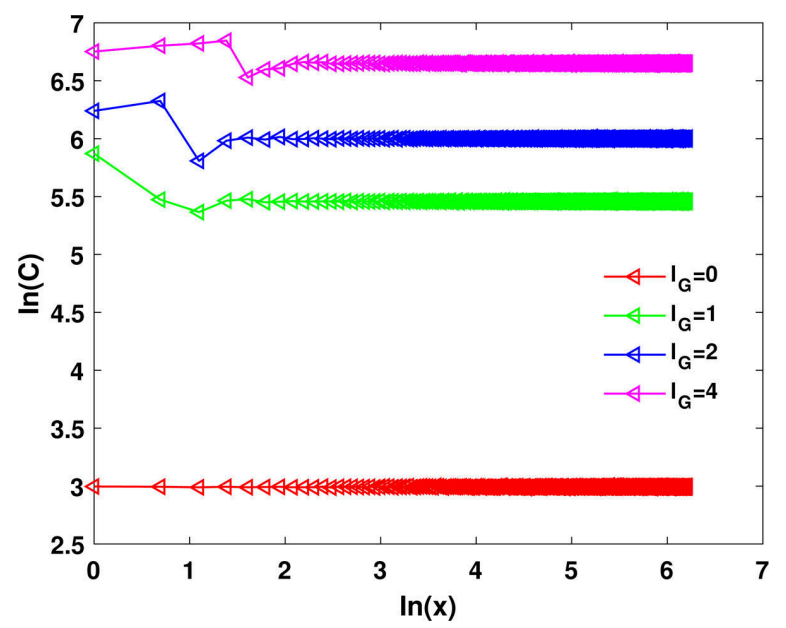

Fig. 6. Correlation in rough surfaces generated by random deposition with system size $L=1000$ and different germination length $l_{G}$ (averages are computed over $k=100$ different configurations).

When $x$ increases, the correlation reaches a constant value that seems independent of the system size $(L)$ but depends on the germination length $\left(l_{G}\right)$.

\section{Conclusions}

This paper proposes a surface growing mechanism able to generate different surface morphologies with a roughness that never saturates. It is based on the fact of introducing a germination length that allows deposited particles to attach to the highest neighbor sites. Thus, the interface density localizes, never saturates and shows a space correlation that depends on that germination length.

\section{References}

Albano, E. V. et al. [1999] "Three-dimensional off-lattice model for the interface growth of polycrystalline materials," Phys. Rev. B 59(11), 7354.

Barabasi, A. L. et al. [1995] Fractal Concepts in Surface Growth (Cambridge University Press).

Barato, A. C. et al. [2008] "Numerical study of a new model for nonequilibrium wetting," Phys. Rev. E 77(1), 011101.

Binder, K. [1976] Monte Carlo Investigations of Phase Transitions and Critical Phenomena (Academic Press).

Cansizoglu, H., et al. [2015] "Investigation of physical vapor deposition techniques of conformal shell coating for core/shell structures by Monte Carlo simulations," Thin Solid Films 583(1), 122-128.

Coy, J. J. and Sidik, S. M. [1985] "Scaling of the active zone in the Eden process on percolation networks and the ballistic deposition model," J. Phys. A, Gen. Phys. 18(2), L75. 
Das Sarma, S. and Tamborenea, P. [1991] "A new universality class for kinetic growth: One-dimensional molecular-beam epitaxy," Phys. Rev. Lett. 66(3), 325.

Duke, C. B. and Plummer, E. W. [2002] Frontiers in Surface and Interface Science (North Holland, Elsevier).

Elsholz, F. and Schöll, E. [2004] "Control of surface roughness in amorphous thin-film growth," Appl. Phys. Lett. 84(21), 4167.

Family, F. [1986] "Scaling of rough surfaces: Effects of surface diffusion," J. Phys. A, Math. Gen. 19(8), L441.

Family, F. and Vicsek, T. [1991] Dynamics of Fractal Surfaces (World Scientific, Singapore).

Forgerini, F. L. and Figueiredo, W. [2009] "Random deposition of particles of different sizes," Phys. Rev. E 79(4), 1602.

Forgerini, F. L. and Figueiredo, W. [2011] "Thin-film growth by random deposition of rod-like particles on a square lattice," Phys. Status Solidi C 8(11), 3119.

Halpin-Heraly, T. and Zhang, Y. C. [1995] "Kinetic roughening phenomena, stochastic growth, directed polymers and all that. Aspects of multidisciplinary statistical mechanics," Phys. Rep. 254(4), 215.

Haselwandter C. A. and Vvedensky D. D. [2007] "Multiscale theory of fluctuating interfaces: Renormalization of atomistic models," Phys. Rev. Lett. 98(4), 046102.

Jeong, S. H. and Boo, J. H. [2004] "Influence of target-to-substrate distance on the properties of AZO films grown by RF magnetron sputtering," Thin Solid Films 447-448, $105-110$.

Majaniemi S. et al. [1996] "Kinetic roughening of surfaces: Derivation, solution, and application of linear growth equations," Phys. Rev. B 53(12), 8071-8082.

Mal, B. et al. [2011] "Revisiting surface diffusion in random deposition," Eur. Phys. J. B $\mathbf{8 2}(3), 341$.

Meakin, P. et al. [1985] "Dynamic cluster-size distribution in cluster-cluster aggregation: Effects of cluster diffusivity," Phys. Rev. B, Condens. Matter 31(1), 564.

Meakin, P. [1993] "The growth of rough surfaces and interfaces," Phys. Rep. 235(4), 189.

Mirabella, D. A. and Aldao, C. M. [2016] "Surface growth by random deposition of rigid and wetting clusters," Surf. Sci. 246, 282-287.

Petrov, I. et al. [2003] "Microstructural evolution during film growth," J. Vac. Sci. Technol. A 21, S117-S128.

Raoufi, D. and Hosseinpanahi, F. [2013] "The effect of film thickness on surface morphology of ITO thin films," J. Theor. Appl. Phys. 7, 21.

Saoudi, A. et al. [2017] "On the interfacial thermal properties of two rough surfaces in contact in preimpregnated composites consolidation," Surf. Topography, Metrol. Prop. $\mathbf{5}(4), 5010$. 